\title{
Assessment of valgus laxity after release of the medial structure in medial open-wedge high tibial osteotomy: an in vivo biomechanical study using quantitative valgus stress radiography
}

Dai Sato ${ }^{1}$, Eiji Kondo ${ }^{2 *}$, Koji Yabuuchi ${ }^{1}$, Jun Onodera ${ }^{3}$, Tomohiro Onodera ${ }^{1}$, Tomonori Yagi ${ }^{3}$, Keita Sakamoto ${ }^{4}$, Akira Takasawa ${ }^{5}$, Norimasa Iwasaki ${ }^{1}$ and Kazunori Yasuda ${ }^{3}$

\begin{abstract}
Background: To perform medial open-wedge high tibial osteotomy (OWHTO), surgeons expose the medialproximal tibia by releasing or cutting the superficial layer of the medial collateral ligament (sMCL). Biomechanically, the $S M C L$ provides primary restraint against valgus forces. Therefore, any release of the $S M C L$ can cause valgus instability of the knee joint. The purpose of this study was to assess valgus laxity after release of the medial structure of the knee during OWHTO.

Methods: Between 2009 and 2015, 84 consecutive patients (93 knees) who underwent OWHTO using a locking plate were enrolled in this study. All patients underwent radiological examinations before surgery, during surgery, 1 year after surgery, and after plate removal to objectively assess valgus laxity. The medial joint space (MJS) and the joint line convergence angle (JLCA) of the knee were evaluated using quantitative valgus stress radiography. Clinical evaluation was performed 2 years after surgery.

Results: The mean functional knee score improved significantly, from 65.5 to 91.1 points $(p<0.0001)$. The mechanical axis percentage shifted to pass through a point $69.7 \%$ lateral from the medial edge of the tibial plateau. The MJS and JLCA increased significantly during OWHTO surgery $\left(11.0 \mathrm{~mm}, 7.4^{\circ}, p<0.0001\right)$. However, no significant differences were noted in the MJS and JLCA among preoperative, 1-year postoperative periods and after plate removal.

Conclusion: Valgus laxity was significantly greater after release of the SMCL. However, no significant differences were noted in valgus laxity in preoperative, 1-year postoperative periods and after plate removal. Complete release of the $S M C L$ did not cause postoperative valgus laxity after OWHTO surgery.
\end{abstract}

Trial registration: Trial registration number: No.012-0360.

Keywords: Medial open-wedge high tibial osteotomy, Superficial layer of the medial collateral ligament, Valgus instability, Valgus stress test

\footnotetext{
* Correspondence: eijik@med.hokudai.ac.jp

${ }^{2}$ Centre for Sports Medicine, Hokkaido University Hospital, Kita-14 Nishi-5,

Kita-ku, Sapporo 060-8648, Japan

Full list of author information is available at the end of the article
}

(c) The Author(s). 2019 Open Access This article is distributed under the terms of the Creative Commons Attribution 4.0 International License (http://creativecommons.org/licenses/by/4.0/), which permits unrestricted use, distribution, and reproduction in any medium, provided you give appropriate credit to the original author(s) and the source, provide a link to the Creative Commons license, and indicate if changes were made. The Creative Commons Public Domain Dedication waiver (http://creativecommons.org/publicdomain/zero/1.0/) applies to the data made available in this article, unless otherwise stated. 


\section{Introduction}

High tibial osteotomy (HTO) has been a useful surgical option for medial osteoarthritis (OA) and spontaneous osteonecrosis of the knee (SONK) $[1,2]$. Recently, medial open-wedge $(\mathrm{OW}) \mathrm{HTO}$ with a locking plate has attracted a great deal of attention [1-9]. To obtain sufficient opening of the medial side of the proximal tibia in OWHTO, surgeons should detach the distal attachment of the superficial layer of the medial collateral ligament (sMCL) or completely cut the sMCL at the osteotomy level [10]. The human MCL consists of three units: the superficial parallel-oriented fibers (sMCL), the deep medial capsular ligament, and the posterior oblique ligament [11-13]. Biomechanically, the sMCL is the primary restraint to valgus forces and plays a significant role in restraining external rotation $[14,15]$. Therefore, detachment or cut of the sMCL induces valgus instability of the knee joint [11, 16-19]. Clinically, it is known that valgus instability of the knee results in a serious disturbance in walking [20]. Even for aged patients, Dragosloveanu et al. [21] reported that repair of collateral ligament injuries must be performed during surgery, especially complete ruptures of the MCL. Therefore, valgus instability of the knee due to detachment or cut of the sMCL is a potential problem in OWHTO, although such valgus instability has not been included in a list of common complications following OWHTO. Pape et al. [19] suggested that the release of the sMCL should be kept to a minimum to decrease the potential for valgus instability following OWHTO, based on their basic biomechanical study.

Most recently, Seo et al. [22] reported the first clinical study to evaluate the changes in valgus laxity of the knee joint after OWHTO with a locking plate using valgus stress radiographs. They reported that complete release of the sMCL increased valgus laxity during surgery, but the laxity decreased to the level before sMCL release at 3 and 12 months. However, they did not measure valgus laxity after removal of the locking plate, which might affect the measured valgus laxity as late instability. In addition, their clinical outcomes were still lacking. Thus, the database on valgus laxity of the knee after OWHTO is still insufficient. Specifically, it remains unclear how much valgus laxity remains after removal of the medial locking plate.

The purpose of present study was to investigate the changes in the valgus laxity of the knee joint after removal of the medially placed locking plate in patients who underwent OWHTO with complete release of the sMCL using quantitative valgus stress radiography. The hypotheses were that (1) release of the distal attachment of the sMCL may significantly increase valgus laxity immediately during surgery; (2) there may be no significant difference in valgus laxity between pre-operative and at 1 year periods after OWHTO with medial locking plate fixation; and (3) removal of the locking late after OWHTO may not significantly increase valgus laxity.

\section{Materials and methods Study design}

Between January 2009 and July 2015, a prospective cohort study was performed with 84 consecutive patients (93 knees) who underwent OWHTO with a locking plate (TomoFix $^{\oplus}$ Medial High Tibial Plate, DepuySynthes, West Chester, PA, USA, or TriS Medial HTO Plate System, Olympus Terumo Biomaterials, Tokyo, Japan). Inclusion criteria involved persistent pain due to medial compartment OA or the SONK of medial femoral condyle. Each patient received conservative treatment for at least 3 months. Exclusion criteria included: (1) lateral femorotibial angle (FTA) greater than $185^{\circ}$; (2) loss of knee extension more than $15^{\circ}$; (3) range of knee motion less than $130^{\circ}$; (4) history of infection in the knee; (5) severe OA in the patellofemoral or/and the lateral femorotibial joints; and (6) cruciate ligament insufficiency or varus/valgus instability of greater than $10^{\circ}$. All patients underwent clinical and radiological examinations before surgery and 2 years after surgery. In addition, 10 patients underwent spot MRI evaluations before surgery and after plate removal. In three patients, a needle biopsy was taken from the distal attachment of the sMCL before surgery and after plate removal.

\section{Patient demographics}

The overall demographic data are summarized in Table 1 . There were 20 men and 64 women with a mean age of 60.4 (range 41-72) years at the time of surgery. Overall, 84 knees were diagnosed as medial compartment OA, and 9 knees were diagnosed as SONK of the medial femoral condyle.

\section{Preoperative planning}

Preoperative planning with an appropriate correction angle of the tibia is made using a standing full-length lower limb anteroposterior radiograph [24]. OW osteotomy lines are drawn on the full-length lower limb radiograph so that the hinge point, $\mathrm{P}$, is located at approximately $5 \mathrm{~mm}$ medial from the tibio-fibular joint on the lateral tibial condyle. To calculate an appropriate angle of the medial opening wedge, a long line, $[\mathrm{A}]$, is drawn from the center of the femoral head through the $65 \%$ lateral from the medial edge of the tibial plateau on the lateral tibial plateau. Then, another line, $[\mathrm{B}]$, is drawn from the hinge point $[\mathrm{P}]$ to the center of the talar dome, and the length of line $\mathrm{B}$ is measured. Then, an arc, $[C]$, the center and the radius of which are the hinge point $\mathrm{P}$ and line $\mathrm{B}$, respectively, is drawn so that the arc is across line $\mathrm{A}$. A line, $[\mathrm{D}]$, is drawn from the hinge point $\mathrm{P}$ to the crossing point between line $\mathrm{A}$ and the arc $\mathrm{C}$. Then, the angle formed between line $\mathrm{B}$ and line $\mathrm{D}$ provides the 
Table 1 Patients' background characteristics

\begin{tabular}{ll}
\hline Variable & Frequency \\
\hline Age (y) & $60.4(8.7)$ \\
Male/female (patients) & $20 / 64$ \\
Right/left side (knees) & $51 / 42$ \\
Unilateral/bilateral (knees) & $84 / 9$ \\
Body weight (kg) & $65.7(12.1)$ \\
BMl (kg/m ${ }^{2}$ ) & $26.6(4.0)$ \\
Young adult mean (\%) of the & $90.8(15.8)$ \\
bone mineral density & \\
OA grade ${ }^{\text {(patients) }}$ & \\
Grade 0 & 0 knees \\
Grade 1 & 0 knees \\
Grade 2 & 17 knees \\
Grade 3 & 61 knees \\
Grade 4 & 6 knees \\
SONK ${ }^{b}$ (patients) & \\
Stage 1 & \\
Stage 2 & 0 knees \\
Stage 3 & 4 knees \\
Stage 4 & 4 knees \\
\hline
\end{tabular}

Mean (standard deviation).

OA osteoarthritis, SONK spontaneous osteonecrosis of the knee.

${ }^{a}$ Kellgren-Lawrence grading system

boshino's stage [23]

medial opening angle, which is identical to the correction angle of the lower limb alignment. A medial opening line from the hinge point $[\mathrm{P}]$ is drawn using this angle. Finally, the medial opening distance is also measured on the medial side of the proximal tibia.

\section{Surgical procedure of OWHTO}

Before OWHTO, arthroscopy was routinely performed to evaluate the cartilages, menisci, and cruciate ligaments. Additional treatments of the intra-articular lesions are shown in Table 2. The OWHTO procedure was recently reported in detail [25]. A 7-cm medial longitudinal incision was made in the proximal tibia. Complete release of the medial structure of the knee was performed, which consisted of the distal attachment of the sMCL, the pes anserinus, and periosteum. Then, an ascending biplanar osteotomy of the tibial tuberosity was

Table 2 Arthroscopic intra-articular treatment

Number of knees

\begin{tabular}{ll}
\hline Partial meniscectomy & \\
Medial meniscus & 69 knees \\
Lateral meniscus & 13 knees \\
Microfracture & 41 knees \\
Osteochondral autograft transfer & 9 knees \\
\hline
\end{tabular}

performed. The oblique osteotomy site was gradually opened for the correction angle and target width using a protractor-installed specially designed spreader (Olympus Terumo Biomaterials) based on preoperative planning. Under fluoroscopic control, the surgeon confirmed that the mechanical axis of the correct lower limb using a long straight metal rod. When the mechanical axis (MA) passed through a point $65 \%$ lateral from the medial edge of the tibial plateau, the wedged $\beta$-tricalcium phosphate spacers (Osferion60, Olympus Terumo Biomaterials) were implanted parallel into the posterior opening space (Fig.1a). Before implantation of the plate, the sMCL, pes anserinus, and periosteum were sutured using absorbable sutures (Fig. 1b). Finally, the tibia was fixed with a locking plate (Fig. 1c) by inserting 8 locking screws (Fig. 1d, e).

\section{Clinical and radiological evaluations}

The patients were evaluated using the functional knee scoring scale (Japanese Orthopaedic Association (JOA) score for osteoarthritic knees) [26, 27]. The clinical evaluations were carried out twice, before surgery and 2 years after OWHTO. Radiographs were obtained both pre-operatively and postoperatively (one and 2 years after OWHTO). The FTA was measured on an anteroposterior weight-bearing radiograph of a single leg with the knee joint in extension. The MA percentages, the hip knee angle (HKA), and the medial proximal tibial angle (MPTA) were measured on an anteroposterior radiograph of the whole lower limb taken with a long cassette in the one-leg standing position. The Insall-Salvati index [28] and posterior tibial slope (PTS) [29] were estimated on lateral radiographs.

\section{Valgus laxity evaluation}

To objectively assess valgus laxity, the joint line convergence angle (JLCA) and the distance of the medial joint space (MJS) were evaluated with a $150-\mathrm{N}$ valgus force at $20^{\circ}$ of knee flexion using telos device (Metax, HungenObbornhofen, Germany). Anteroposterior stress radiographs were obtained five times: 1 ) before surgery; 2) before releasing the distal attachment of the SMCL under general anesthesia; 3) after releasing the sMCL; 4) 1 year after OWHTO surgery; and 5) 2 years after OWHTO surgery (after plate removal). Then, intraoperative valgus stress radiographs were performed using a knee laxity tester (Kalamazoo, MI, USA). The distance of the MJS [30] was calculated according to the method reported by Sawant et al. (Fig. 2). The JLCA [31] was calculated according to the method reported by Lee et al. (Fig. 2).

\section{MRI evaluation}

Ten patients underwent spot MRI evaluations before surgery and after plate removal. MRI was performed 


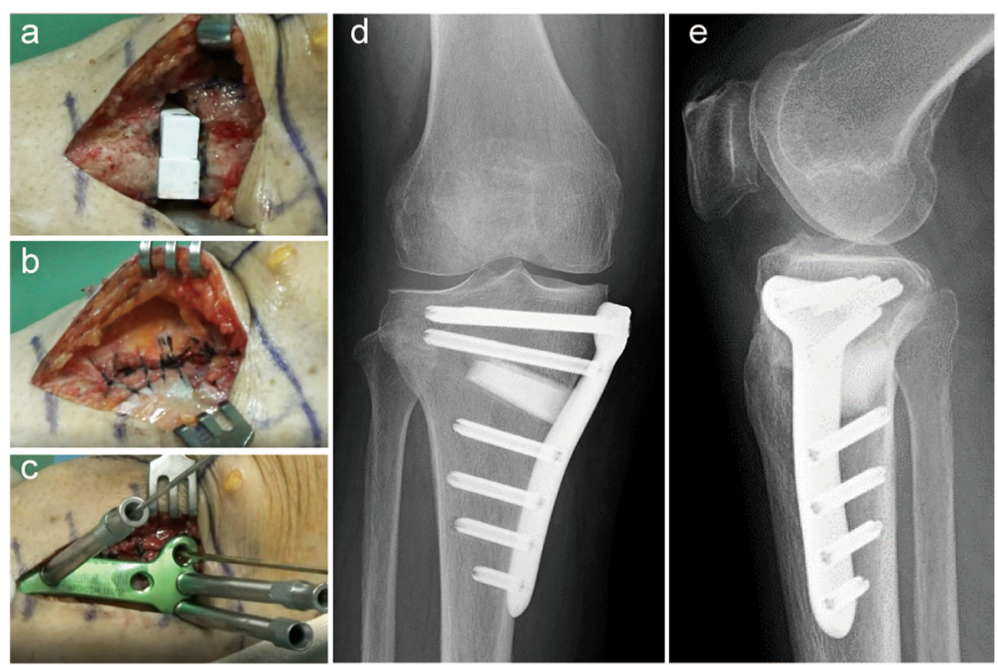

Fig. 1 a Equivalent to the size of the opening, the wedged $\beta$-tricalcium phosphate spacers (Osferion60, Olympus Terumo Biomaterials) are inserted into the osteotomy site to fit into the medial cortical bone edge. $\mathbf{b} \mathrm{SMCL}$, pes anserinus, and periosteum are repaired. $\mathbf{c}$ The locking plate (TriS Medial HTO Plate System, Olympus Terumo Biomaterials) is fixed on the medial aspect of the proximal tibia. Postoperative anteroposterior (d) and lateral (e) radiographs of the right knee show a low-profile locking plate (TriS Medial HTO Plate System, Olympus Terumo Biomaterials) fixed in place with eight locking screws

using a 1.5-Tesla whole-body clinical scanner (Achieva TX-series; Philips Healthcare, Best, The Netherlands). The distal attachment of the sMCL of each subject was centered in a microscopy coil in the supine position with the knee slightly flexed. Two-plane (coronal and sagittal) localizer imaging was performed for the distal attachment of the sMCL. The parameters for this imaging included: repetition time (TR), $4000-8000 \mathrm{~ms}$; echo time (TE), $70 \mathrm{~ms}$; matrix size, $256 \times 256$; slice thickness, 2 $\mathrm{mm}$; and number of 16 slices.

\section{Histological evaluation}

Samples of the distal attachment of the sMCL were taken to use a biopsy needle. The samples were fixed with $10 \%$ formalin, dehydrated, embedded in paraffin, and sliced into 4- $\mu \mathrm{m}$-thick sections stained with hematoxylin and eosin (H-E), Masson trichrome, and Elastica van Gieson (EVG).

\section{Statistical analysis}

All data are shown as means with standard deviations. A commercially available software program (GraphPad Software, Inc., San Diego, CA, USA) was used for the statistical calculations. The paired $t$-test was used to assess the clinical and radiological differences between before and after surgery. Repeated-measures analysis of variance (ANOVA) was used to compare valgus instability among the five groups: 1) before surgery; 2) before releasing the proximal tibial attachment the sMCL under general anesthesia; 3) after releasing the proximal tibial attachment of the sMCL; 4) 1 year after surgery; and 5)
2 years after surgery (after plate removal). To identify which preoperative, intraoperative, and postoperative factors affect knee laxity in the valgus stress test and other related outcomes, Pearson correlation analysis was used. The significance level was set at $p=0.05$.

\section{Results}

\section{Overall clinical and radiological outcomes}

The mean opening angle and distance were $10.1 \pm 3.5^{\circ}$ and $11.9 \pm 2.6 \mathrm{~mm}$. The mean JOA score improved significantly from 65.5 points to 91.1 points (Table 3 ). The mean FTA and HKA changed significantly from $179.6^{\circ}$ to $169.6^{\circ}$ and from $-4.5^{\circ}$ to $3.7^{\circ}$. The mean MA shifted to a point $69.7 \%$ lateral from the medial edge of the tibial plateau. The mean MPTA and tibial slope angle were significantly increased from $85.5^{\circ}$ to $93.3^{\circ}$ and from $8.5^{\circ}$ to $11.0^{\circ}$.

\section{Analysis of valgus laxity}

Comparisons of MJS and JLCA among 5 periods are presented in Fig. 3a, b. The MJS and JLCA before releasing the medial structure under general anesthesia was significantly increased compared with the MJS and JLCA before surgery $(6.4 \pm 1.7 \mathrm{~mm}$ to $8.0 \pm 3.7 \mathrm{~mm}$ and $-0.5 \pm$ $2.0^{\circ}$ to $4.3 \pm 1.9^{\circ}$ ). In addition, the MJS and JLCA after release of the medial structure during surgery was significantly increased $(6.4 \pm 1.7 \mathrm{~mm}$ to $11.0 \pm 4.5 \mathrm{~mm}$ and $-0.5 \pm 2.0^{\circ}$ to $\left.7.4 \pm 2.7^{\circ}\right)$. However, there were no significant differences in the MJS and JLCA among the three periods before surgery, 1 year after OWHTO surgery, and after plate removal (Fig. 3). The correlation 


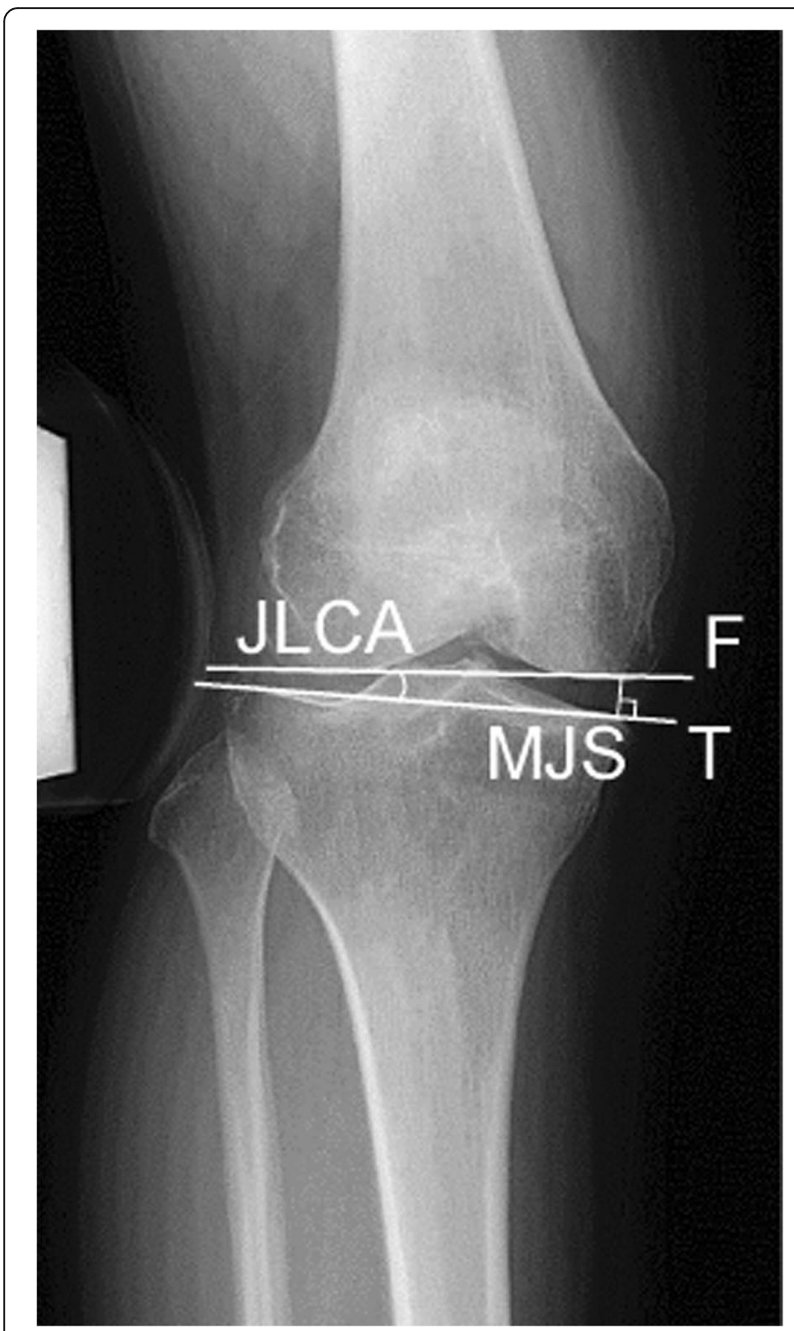

Fig. 2 Measurement of the medial joint space (MJS) and joint line convergence angle (JLCA)

Table 3 Comparisons of clinical and radiological outcomes between pre-operative and 2 years postoperative

\begin{tabular}{llll}
\hline & Pre-operative & 2 years postoperative & $p$ value $^{a}$ \\
\hline $\begin{array}{l}\text { JOA score } \\
\text { (points) }\end{array}$ & $65.5(10.1)$ & $91.1(8.3)$ & $<0.0001$ \\
FTA $\left(^{\circ}\right)$ & $179.6(3.3)$ & $169.6(2.3)$ & $<0.0001$ \\
HKA $\left(^{\circ}\right)$ & $-4.5(2.9)$ & $3.7(1.9)$ & $<0.0001$ \\
MA $(\%)$ & $27.4(12.6)$ & $69.7(13.0)$ & $<0.0001$ \\
MPTA $\left(^{\circ}\right)$ & $85.5(2.8)$ & $93.3(2.5)$ & $<0.0001$ \\
Insall-Salvati & $0.9(0.1)$ & $0.9(0.1)$ & N.S. \\
ratio $(\%)$ & & $11.0(3.4)$ & $<0.0001$ \\
PTS $\left(^{\circ}\right)$ & $8.5(3.2)$ & &
\end{tabular}

JOA score Japanese Orthopaedic Association score for osteoarthritic knee, FTA lateral femorotibial angle, HKA hip knee angle, MA mechanical axis, MPTA medial proximal tibial angle, PTS posterior tibial slope.

${ }^{a}$ Significance levels with Student's $t$-test analysis demonstrated that the MJS and JLCA before surgery were moderately correlated and significantly more than those after sMCL release $(p<0.0180)$ and after plate removal $(p<0.0001)$ (Fig. 4$)$. The regression analysis showed moderate correlation between $\triangle \mathrm{JLCA}$ (preoperative JLCA - postoperative JLCA) and overcorrection (postoperative MA - 65\%) [correlation coefficient (R), 0.4338] (Fig. 5).

\section{MRI evaluation}

Spot MRI showed that the sMCL was clearly seen as regular in shape before OWHTO surgery. On the other hand, after OWHTO, the MRI showed that the sMCL was meandering and seen to be thicker with isointensity compared to before surgery (Fig. 6).

\section{Histological evaluation}

In the normal control, $\mathrm{H}-\mathrm{E}$ staining showed many cell nuclei in the distal attachment of the sMCL (Fig. 7a). Masson trichrome and EVG staining showed intact elastic fibers in the bone in the sMCL (Fig. 7b, c). On the other hand, after HTO surgery, H-E staining showed signs of necrotic cell nuclei in the sMCL and bone (Fig. $7 d)$. The bone-ligament interface was filled with unstructured fibrous connective tissue. On Masson trichrome and EVG staining, collagen fiber bundles entering into the bone substrate were irregularly oriented (Fig. 7e, f).

\section{Discussion}

The most important findings of the present study were that: (1) the release of the distal attachment of the sMCL significantly increased valgus laxity immediately during OWHTO surgery; (2) there was no significant difference in the valgus laxity between the pre-operative and at 1 year periods after OWHTO with medial locking plate fixation; and (3) removal of the locking plate after OWHTO did not significantly increase valgus laxity. These results suggest that release of the distal attachment of the sMCL may not cause valgus laxity after OWHTO surgery.

In several biomechanical studies, release of the SMCL is essential for osteotomy procedure when performing OWHTO $[2,10,19,32]$. There studies also reported that this procedure is necessary to avoid not only an increase in medial joint pressure and posterior slope, but also neurovascular injury by inserting a protector to the posterior tibia during surgery. First, decompression of the medial compartment, as the main goal of OWHTO surgery, cannot be achieved if the sMCL remains intact, because the medial opening space is created between the proximal and the distal attachment of the SMCL. Agneskirchner et al. [10] tested human knee specimens with a load of $1000 \mathrm{~N}$ with different loading alignments in extension using a material testing machine. They 

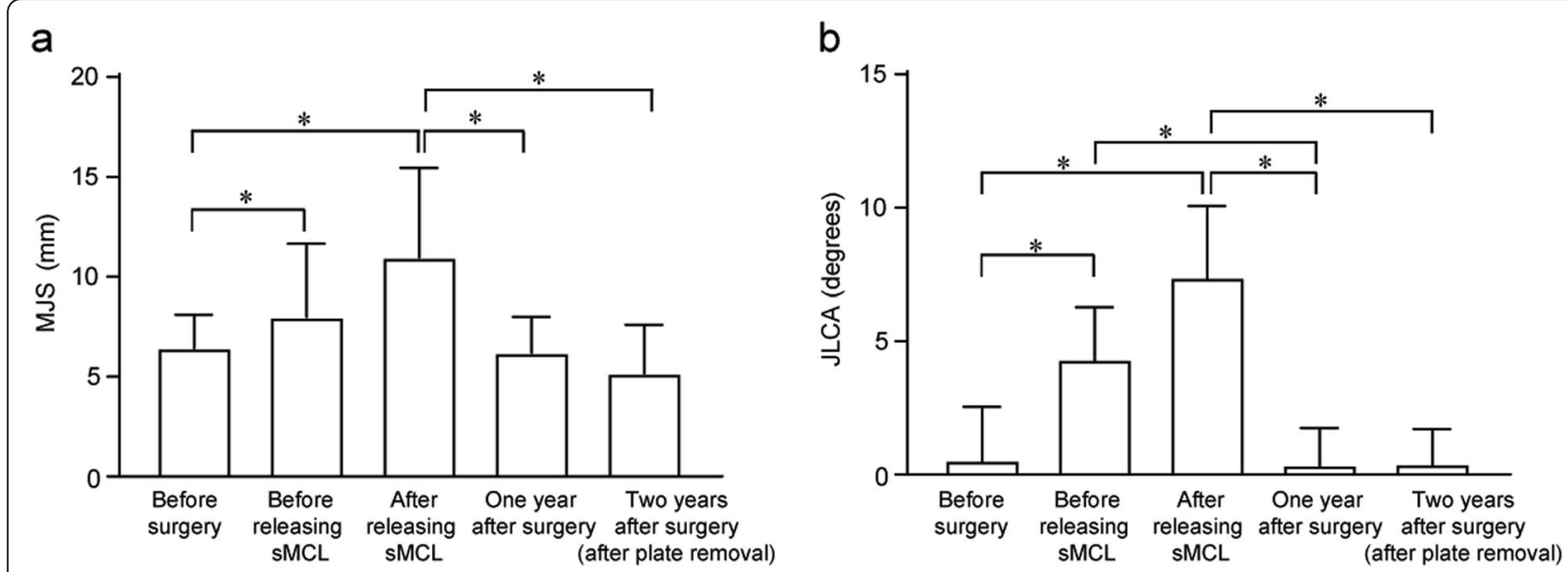

Fig. 3 Changes in the medial joint space (MJS) (a) and in the joint line convergence angle (JLCA) (b) in all patients. Error bars represent the standard deviation. ${ }^{*}$ Statistically significant difference $(p<0.05)$

concluded that complete release of the distal fibers of the SMCL is necessary to decompress medial joint pressure after OWHTO. Second, the tibial slope increase in OWHTO was caused by asymmetric opening of the oblique osteotomy site [2]. If the osteotomy is started in the medial tibial head (anterior to the MCL), the osteotomy is too short in the posterior part of the tibia. Then, the posterior part of the tibial plateau is unavoidably lifted less than the anterior part, and the slope is thus increased. Therefore, the sMCL should be released to avoid increasing the tibial slope in OWHTO, as reported by Lobenhoffer et al. [2]. Third, protection for the posterior neurovascular structure is necessary during wedge osteotomy from the medial cortex of the tibia to the proximal tibiofibular joint in OWHTO. Previous studies reported that the frequency of popliteal artery injury was 0.4-1.7\% during OWHTO [33-35]. Attinger et al. [32] reported that sufficient medial exposure for orientation a

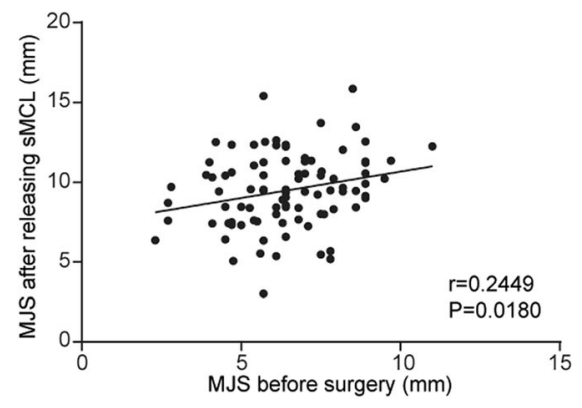

C

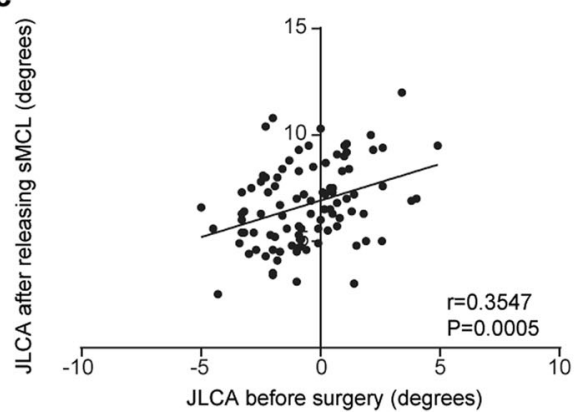

b

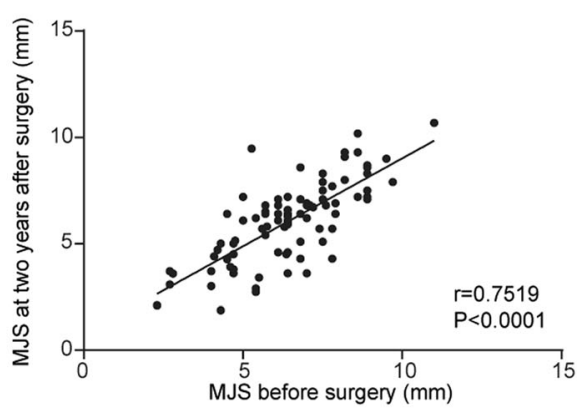

d

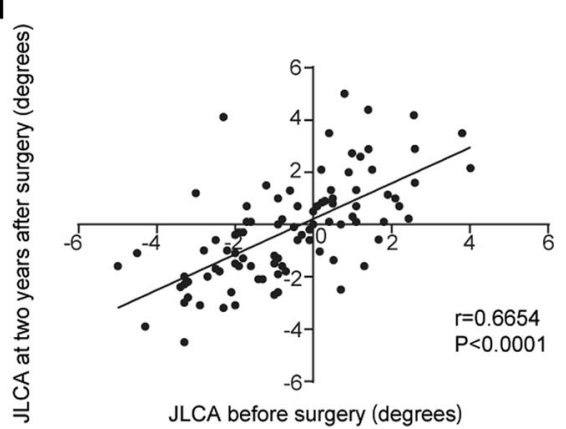

Fig. 4 Pearson correlation analysis shows that the MJS and JLCA before surgery are significantly correlated with the MJS $(r=-0.2449, p=0.0180)$ and JLCA $(r=-0.3547, p=0.0005)$ after releasing the SMCL $(\mathbf{a}, \mathbf{c})$ and the MJS $(r=-0.7519, p<0.0001)$ and JLCA $(r=0.6654, p<0.0001)$ after plate removal (2 years after OWHTO) $(\mathbf{b}, \mathbf{d})$ 


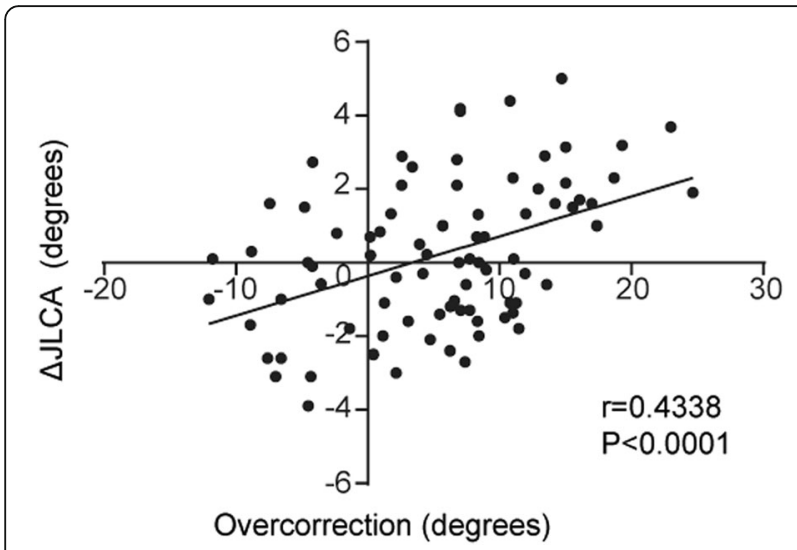

Fig. 5 The relationship between $\triangle \mathrm{LCCA}$ and overcorrection. The scatter plot shows a moderate correlation between $\triangle \mathrm{LCCA}$ and overcorrection $(R=0.4338)$

and protection by releasing the SMCL during OWHTO surgery is mandatory for a safe osteotomy. In their report, it was easy to insert a blunt retractor to protect the popliteal artery when releasing the sMCL.

Previous basic studies reported that the SMCL is the 'primary' restraint to valgus forces $[11,13,15,18,19,36]$. Seo et al. [22] reported that a statistically significant difference was observed between the MJS before (mean; 9.0 $\mathrm{mm})$ and after release of the sMCL $(12.2 \mathrm{~mm})$ during OWHTO surgery. Pape et al. [19] conducted a cadaveric study on medial joint opening after release of the sMCL. According to their results, the average increase was 3.9 $\mathrm{mm}$ after complete release of the $\mathrm{sMCL}$ and $3.6 \mathrm{~mm}$ after partial release. These increase values $(3 \mathrm{~mm})$ were similar to those of the present study. Seo et al. [22] noted that the MJS after fixation with a plate following opening of the osteotomy site $(9.2 \mathrm{~mm})$ was significantly decreased compared with the MJS after release of the sMCL $(12.2 \mathrm{~mm})$. We speculated as to the reasons why valgus laxity after complete release of the sMCL was significantly decreased by OWHTO. The first reason is tensioning of medial structures (pes anserinus and semimembranosus tendon) other than the sMCL due to medial opening. The second is medial fixation of the locking plate. The third is recovery of valgus laxity after healing of the sMCL after OWHTO. However, Seo et al. did not measure valgus laxity after removal of the locking plate.

OWHTO surgery was performed aiming to MA passing through $65 \%$ point lateral from the medial edge of the tibial plateau. However, the mean MA was $69.7 \%$ after 2 years. We considered this discrepancy between intra- and post-operative MA in the present study. First, in the OWHTO procedures, intra-operative assessments of alignment have been performed under non-weight bearing conditions with the patient in the supine position. Therefore, discrepancies have arisen between intra-operative prediction of alignment correction and real alignment assessed in standing patients by lower limb radiographs. Second, we have focused on the difference of pre- and postoperative JLCA, which was associated with greater overcorrection of lower limb alignment [31]. Previous study [37] reported that $\triangle$ JLCA was significantly correlated with overcorrection. In the present study, the regression analysis also showed moderate correlation between $\triangle \mathrm{JLCA}$ and overcorrection. From these results, we speculated that amount of change from pre- to postoperative JLCA has affected discrepancies between intra- and postoperative alignment after OWHTO.

Pape et al. [19] reported that complete release of the sMCL during OWHTO has the potential for valgus instability of the knee joint in their biomechanical study. In particular, they mentioned the potential for late valgus instability following OWHTO [19]. Clinical problems regarding valgus instability after OWHTO surgery are very few. However, recently, Kim et al. [38] reported two patients who underwent conversion to total knee arthroplasty (TKA) using a constrained-type implant after OWHTO due to neglected valgus instability. The anatomical changes in the proximal tibia after OWHTO may cause soft tissue imbalance. After OWHTO surgery,
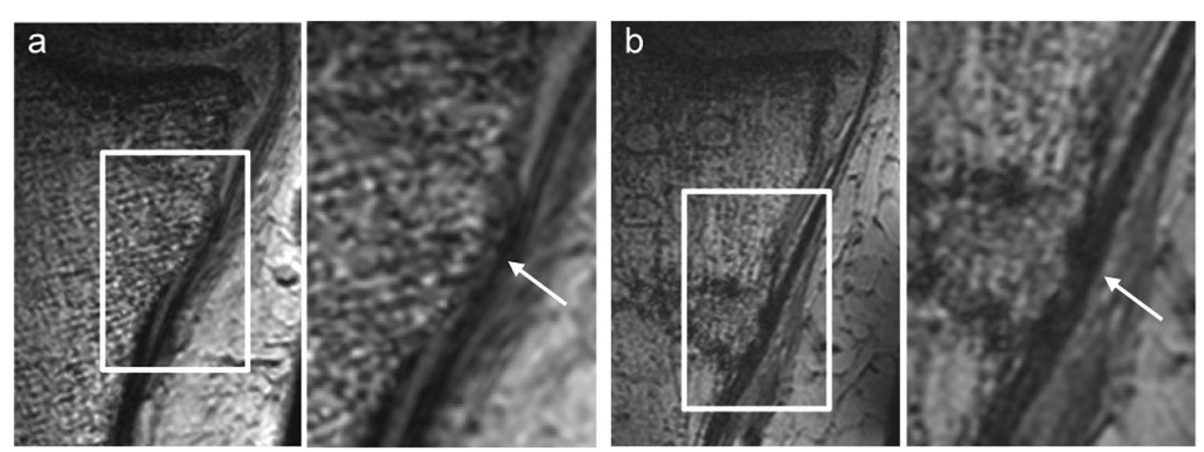

Fig. 6 a Preoperative T2-weighted coronal plane MRI showing the SMCL. b After removal of a locking plate, the sMCL (indicated by white arrows) shows iso-intensity and is thicker than on the preoperative image 

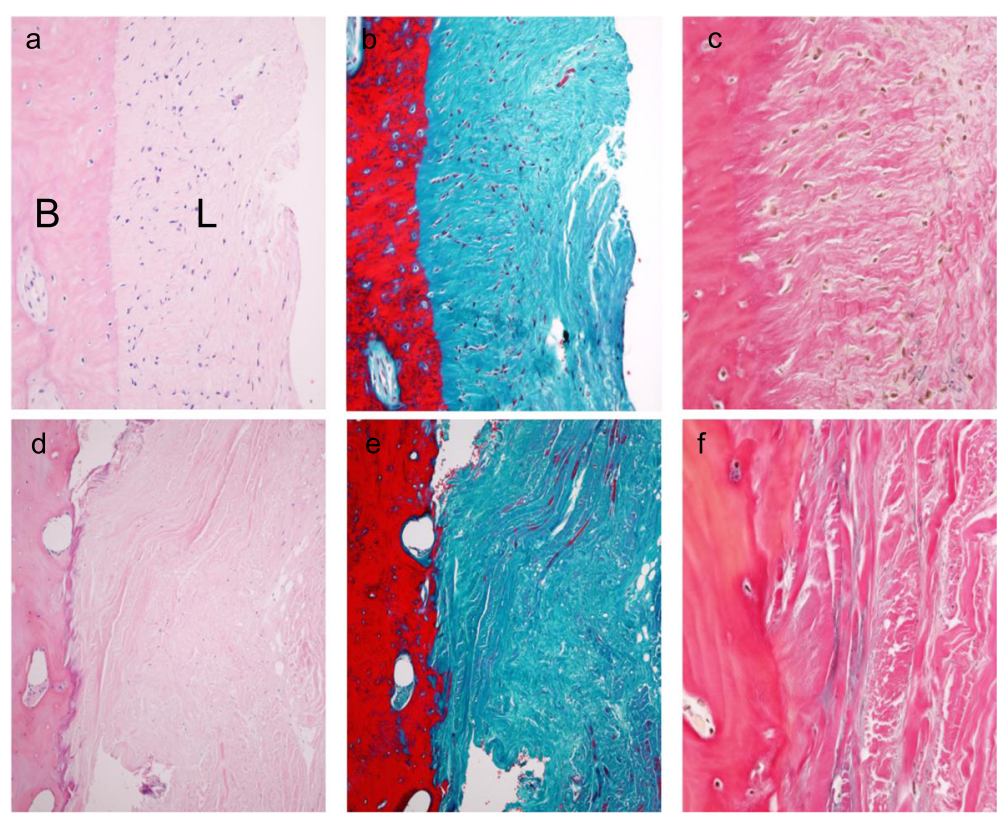

Fig. 7 Images showing the histomorphometry using hematoxylin-eosin (left), Masson trichrome (middle) and Elastica van Gieson (right) staining of the distal attachment of the $\mathrm{SMCL}$ in the normal control $\mathbf{a}, \mathbf{b}, 100 x$ magnified, $\mathbf{c} 400 x$ magnified. After removal of a locking plate $\mathbf{d}$, e, 100x magnified, e 400x magnified. L: ligament B: bone

the medial tibial plateau is positioned higher than the lateral tibial plateau. If the tibial bone cutting is performed perpendicular to the mechanical axis, it can cause large resection of medial tibial bone that may result in valgus instability and thus increase the risk of soft tissue imbalance. In addition, soft tissue balance should also be considered when performing TKA after OWHTO. Valgus instability can also occur after plate removal during TKA conversion due to the required medial release. Because the distal attachment of the sMCL is usually released in OWHTO, deep MCL release in converting to TKA can affect the biomechanical function of the sMCL, which can cause valgus instability. Therefore, the elevated distal portion of the sMCL should be placed in situ on the graft bone, and pes anserinus, if released, should be repaired during OWHTO to prevent valgus instability. Many surgeons usually release the sMCL subperiosteally distal to the osteotomy site or completely 'cut' it at the osteotomy site without repair when performing OWHTO. Therefore, the valgus instability could be the result of the previous OWHTO surgeries.

In the present study, it was noted that complete release of the distal attachment of the sMCL did not cause postoperative valgus instability. There are several reasons why postoperative valgus instability was not observed 2 years after complete release of the MCL during OWHTO. When no bone graft or bone substitute is placed in the osteotomy site in an OWHTO procedure, it is difficult to repair the released SMCL due to keeping the opening space using a lamina spreader, the locking plate is fixed to the medial side of the proximal tibia with screws. In our surgical procedure, the $\beta$-TCP spacer was implanted into the opening space. Before locking plate fixation of the tibia, the released SMCL and periosteum above the opening space were repaired. Thus, the medial opening space was covered as possible by the SMCL and periosteum. We speculate that the recovery of valgus laxity might be related to this repair procedure. Although the tension of the sMCL might be increased by the repair, we expected that proximally advancing the SMCL from the original position could prevent increasing medial joint pressure of the knee. Seo et al. [22] also reported that valgus laxity of the knee joint was not observed after OWHTO as in the present study. They concluded that valgus laxity induced by the complete release of the SMCL can be recovered through the tension of the medial structure opening the osteotomy site. These could return the valgus stability level to that before the release of the SMCL. A locking plate is thought to be an important factor to stabilize the knee joint after OWHTO surgery [39]. The present study showed that there were no significant differences in valgus laxity between before surgery and after locking plate removal at 2 years after surgery. However, MRI and histological examinations showed that unstructured and irregularly oriented fibrous tissues were observed in the distal attachment of the sMCL.

There are several limitations to this study. First, the follow-up valgus stress radiography was performed 2 
years after surgery. Therefore, at the present time, we cannot speculate on the long-term effect of complete release of the sMCL on valgus stability. Second, the effect of releasing pes anserinus, that is the attachment of the sartorius, gracilis and semitendinosus muscles, was not assessed. Third, it was not clear when the valgus laxity recovered. Stress tests were not performed from after OWHTO surgery to before 1 year after surgery. Fourth, medial joint pressure after tensioning of medial structures due to medial opening was not performed. Therefore, further studies are needed to objectively examine the effects on valgus stability after OWHTO. Fifth, the number of MRI and histological evaluations was limited, which may have caused significant bias.

\section{Conclusion}

This study demonstrated that complete release of the sMCL during OWHTO surgery did not cause postoperative valgus laxity 2 years after the OWHTO procedure.

\section{Abbreviations}

JLCA: Joint line convergence angle; MJS: Medial joint space; MRI: Magnetic resonance imaging; OWHTO: Medial open-wedge high tibial osteotomy; sMCL: Superficial layer of the medial collateral ligament

\section{Acknowledgements}

Not applicable.

\section{Authors' contributions}

Study design: EK. Study conduct: DS, EK, KY, JO, TO, KS, AT, and KT. Data interpretation: DS, EK, JO, TY NI, and KY. Drafting manuscript: DS, EK, and KY. EK takes responsibility for the integrity of the data analysis. All authors have read and approved the final manuscript.

\section{Funding}

This study did not have any grants or funding despite the academic setting of the institutions and authors.

\section{Availability of data and materials}

The datasets used and analyzed in the current study are available from the corresponding author on reasonable request.

\section{Ethics approval and consent to participate}

This study was approved by the institutional review board of Hokkaido University (No.012-0360). All procedures performed in studies involving human participants were in accordance with the ethical standards of the institutional and/or national research committee and with the 1964 Helsinki declaration and its later amendments or comparable ethical standards. Written informed consent was obtained from all individual participants included in the study.

\section{Consent for publication}

Not applicable.

\section{Competing interests}

EK has the following financial relationships to disclose: grant/research funding from Muto Co Ltd. Japan, Smith \& Nephew Endoscopy Japan, Olympus Terumo Biomaterials Japan, and Yufu Itonaga Co Ltd. Japan. All other authors declare that they have no conflict of interest.

\section{Author details}

${ }^{1}$ Department of Orthopedic Surgery, Faculty of Medicine and Graduate School of Medicine, Hokkaido University, Kita-15 Nishi-7, Kita-ku, Sapporo 060-8638, Japan. ${ }^{2}$ Centre for Sports Medicine, Hokkaido University Hospital, Kita-14 Nishi-5, Kita-ku, Sapporo 060-8648, Japan. ${ }^{3}$ Department Orthopedic
Surgery, Yagi Orthopedic Hospital, 1-35, Nishino-3-5, Nishi-ku, Sapporo 063-0033, Japan. ${ }^{4}$ Department of Diagnostic and Interventional Radiology, Hokkaido University Hospital, Kita-14 Nishi-5, Kita-ku, Sapporo 060-8648, Japan. ${ }^{5}$ Department of Pathology, Sapporo Medical University School of Medicine, S-1 W-17, Chuo-ku, Sapporo 060-8556, Japan.

Received: 28 May 2019 Accepted: 30 September 2019

Published online: 27 October 2019

\section{References}

1. Staubli AE, Simoni CD, Babst R, Lobenhoffer P. TomoFix: a new LCP-concept for open wedge osteotomy of the medial proximal tibia - early results in 92 cases. Injury. 2003;34:55-62.

2. Lobenhoffer P, Agneskirchner JD. Improvements in surgical technique of valgus high tibial osteotomy. Knee Surg Sports Traumatol Arthrosc. 2003; 11(3):132-8.

3. Lobenhoffer P, Agneskirchner J. Zoch W: [open valgus alignment osteotomy of the proximal tibia with fixation by medial plate fixator]. Der Orthopade. 2004;33(2):153-60.

4. Amis AA. Biomechanics of high tibial osteotomy. Knee Surg Sports Traumatol Arthrosc. 2013;21(1):197-205.

5. Bonnin MP, Laurent JR, Zadegan F, Badet R, Pooler Archbold HA, Servien E. Can patients really participate in sport after high tibial osteotomy? Knee Surg Sports Traumatol Arthrosc. 2013;21(1):64-73.

6. Floerkemeier S, Staubli AE, Schroeter S, Goldhahn S, Lobenhoffer P. Outcome after high tibial open-wedge osteotomy: a retrospective evaluation of 533 patients. Knee Surg Sports Traumatol Arthrosc. 2013;21(1):170-80.

7. Kohn L, Sauerschnig M, Iskansar S, Lorenz S, Meidinger G, Imhoff AB, Hinterwimmer S. Age does not influence the clinical outcome after high tibial osteotomy. Knee Surg Sports Traumatol Arthrosc. 2013;21(1):146-51.

8. McNamara I, Birmingham TB, Fowler PJ, Giffin JR. High tibial osteotomy: evolution of research and clinical applications--a Canadian experience. Knee Surg Sports Traumatol Arthrosc. 2013;21(1):23-31.

9. Smith JO, Wilson AJ, Thomas NP. Osteotomy around the knee: evolution, principles and results. Knee Surg Sports Traumatol Arthrosc. 2013;21(1):3-22.

10. Agneskirchner JD, Hurschler C, Wrann CD, Lobenhoffer $P$. The effects of valgus medial opening wedge high tibial osteotomy on articular cartilage pressure of the knee: a biomechanical study. Arthroscopy. 2007;23(8):852-61.

11. Grood ES, Noyes FR, Butler DL, Suntay WJ. Ligamentous and capsular restraints preventing straight medial and lateral laxity in intact human cadaver knees. J Bone Joint Surg Am. 1981;63(8):1257-69.

12. Hughston JC, Andrews JR, Cross MJ, Moschi A. Classification of knee ligament instabilities. Part I. the medial compartment and cruciate ligaments. J Bone Joint Surg Am. 1976:58(2):159-72.

13. Hughston JC, Eilers AF. The role of the posterior oblique ligament in repairs of acute medial (collateral) ligament tears of the knee. J Bone Joint Surg Am. 1973;55(5):923-40.

14. Hughston JC. A surgical approach to the medial and posterior ligaments of the knee. Clin Orthop Relat Res. 1973;91:29-33.

15. Griffith CJ, LaPrade RF, Johansen S, Armitage B, Wijdicks C, Engebretsen L. Medial knee injury: part 1, static function of the individual components of the main medial knee structures. Am J Sports Med. 2009;37(9):1762-70.

16. Kennedy JC, Fowler PJ. Medial and anterior instability of the knee. An anatomical and clinical study using stress machines. J Bone Joint Surg Am. 1971:53(7):1257-70.

17. Warren LA, Marshall JL, Girgis F. The prime static stabilizer of the medical side of the knee. J Bone Joint Surg Am. 1974;56(4):665-74.

18. Matsumoto H, Suda Y, Otani T, Niki Y, Seedhom BB, Fujikawa K. Roles of the anterior cruciate ligament and the medial collateral ligament in preventing valgus instability. J Ortho Sci. 2001;6(1):28-32.

19. Pape D, Duchow J, Rupp S, Seil R, Kohn D. Partial release of the superficial medial collateral ligament for open-wedge high tibial osteotomy. A human cadaver study evaluating medial joint opening by stress radiography. Knee Surg Sports Traumatol Arthrosc. 2006;14(2):141-8.

20. Tsukiyama H, Kuriyama S, Kobayashi M, Nakamura S, Furu M, Ito H, Matsuda S. Medial rather than lateral knee instability correlates with inferior patient satisfaction and knee function after total knee arthroplasty. Knee. 2017;24(6): 1478-84.

21. Dragosloveanu S, Cristea S, Stoica C, Dragosloveanu C. Outcome of iatrogenic collateral ligaments injuries during total knee arthroplasty. Eur J Orthop Surg Traumatol. 2014;24(8):1499-503. 
22. Seo SS, Kim CW, Seo JH, Kim DH, Lee CR. Does superficial medial collateral ligament release in open-wedge high Tibial osteotomy for Varus osteoarthritic knees increase Valgus laxity? Am J Sports Med. 2016;44(4):908-15.

23. Koshino T. The treatment of spontaneous osteonecrosis of the knee by high tibial osteotomy with and without bone-grafting or drilling of the lesion. J Bone Joint Surg Am. 1982;64(1):47-58.

24. Miniaci A, Ballmer FT, Ballmer PM, Jakob RP. Proximal tibial osteotomy. A new fixation device. Clin Ortho Related Res. 1989;246:250-9.

25. Onodera J, Kondo E, Omizu N, Ueda D, Yagi T, Yasuda K. Beta-tricalcium phosphate shows superior absorption rate and osteoconductivity compared to hydroxyapatite in open-wedge high tibial osteotomy. Knee Surg Sports Traumatol Arthrosc. 2014;22(11):2763-70.

26. Yasuda K, Majima T, Tsuchida T, Kaneda K. A ten- to 15-year follow-up observation of high tibial osteotomy in medial compartment osteoarthrosis. Clin Orthop Relat Res. 1992;282:186-95.

27. Aoki Y, Yasuda K, Mikami S, Ohmoto H, Majima T, Minami A. Inverted Vshaped high tibial osteotomy compared with closing-wedge high tibial osteotomy for osteoarthritis of the knee. Ten-year follow-up result. J Bone Joint Surg Br. 2006;88(10):1336-40.

28. Insall J, Salvati E. Patella position in the normal knee joint. Radiology. 1971; 101(1):101-4.

29. Moore TM, Harvey JP Jr. Roentgenographic measurement of tibial-plateau depression due to fracture. J Bone Joint Surg Am. 1974;56(1):155-60.

30. Sawant M, Narasimha Murty A, Ireland J. Valgus knee injuries: evaluation and documentation using a simple technique of stress radiography. Knee. 2004;11(1):25-8

31. Lee DH, Park SC, Park HJ, Han SB. Effect of soft tissue laxity of the knee joint on limb alignment correction in open-wedge high tibial osteotomy. Knee Surg Sports Traumatol Arthrosc. 2016;24(12):3704-12.

32. Attinger MC, Behrend H, Jost B. Complete rupture of the popliteal artery complicating high tibial osteotomy. J Orthop. 2014;11(4):192-6.

33. Georgoulis AD, Makris CA, Papageorgiou CD, Moebius UG, Xenakis T, Soucacos PN. Nerve and vessel injuries during high tibial osteotomy combined with distal fibular osteotomy: a clinically relevant anatomic study. Knee Surg Sports Traumatol Arthrosc. 1999;7(1):15-9.

34. Hernigou P, Ma W. Open wedge tibial osteotomy with acrylic bone cement as bone substitute. Knee. 2001;8(2):103-10

35. Woodacre T, Ricketts M, Evans JT, Pavlou G, Schranz P, Hockings M, Toms A. Complications associated with opening wedge high tibial osteotomy-a review of the literature and of 15 years of experience. Knee. 2016;23(2):276-82.

36. Robinson JR, Bull AM, Thomas RR, Amis AA. The role of the medial collateral ligament and posteromedial capsule in controlling knee laxity. Am J Sports Med. 2006;34(11):1815-23.

37. Ogawa H, Matsumoto K, Ogawa T, Takeuchi K, Akiyama H. Preoperative varus laxity correlates with overcorrection in medial opening wedge high tibial osteotomy. Arch Orthop Trauma Surg. 2016;136(10):1337-42.

38. Kim HJ, Kim YG, Min SG, Kyung HS. Total knee arthroplasty conversion after openwedge high tibial osteotomy: a report of three cases. Knee. 2016;23(6):1164-7.

39. Takeuchi R, Ishikawa H, Aratake M, Bito H, Saito I, Kumagai K, Akamatsu Y, Saito T. Medial opening wedge high tibial osteotomy with early full weight bearing. Arthroscopy. 2009;25(1):46-53.

\section{Publisher's Note}

Springer Nature remains neutral with regard to jurisdictional claims in published maps and institutional affiliations.

\section{Ready to submit your research? Choose BMC and benefit from:}

- fast, convenient online submission

- thorough peer review by experienced researchers in your field

- rapid publication on acceptance

- support for research data, including large and complex data types

- gold Open Access which fosters wider collaboration and increased citations

- maximum visibility for your research: over $100 \mathrm{M}$ website views per year

At $\mathrm{BMC}$, research is always in progress.

Learn more biomedcentral.com/submissions 